\title{
Apparent Transition Behavior of Widely-Used Turbulence Models
}

\author{
Christopher L. Rumsey* \\ NASA Langley Research Center, Hampton, VA 23681-2199, USA
}

\begin{abstract}
The Spalart-Allmaras and the Menter SST $k-\omega$ turbulence models are shown to have the undesirable characteristic that, for fully turbulent computations, a transition region can occur whose extent varies with grid density. Extremely fine two-dimensional grids over the front portion of an airfoil are used to demonstrate the effect. As the grid density is increased, the laminar region near the nose becomes larger. In the SpalartAllmaras model this behavior is due to convergence to a laminar-behavior fixed point that occurs in practice when freestream turbulence is below some threshold. It is the result of a feature purposefully added to the original model in conjunction with a special trip function. This degenerate fixed point can also cause nonuniqueness regarding where transition initiates on a given grid. Consistent fully turbulent results can easily be achieved by either using a higher freestream turbulence level or by making a simple change to one of the model constants. Two-equation $k-w$ models, including the SST model, exhibit strong sensitivity to numerical resolution near the area where turbulence initiates. Thus, inconsistent apparent transition behavior with grid refinement in this case does not appear to stem from the presence of a degenerate fixed point. Rather, it is a fundamental property of the $k-\omega$ model itself, and is not easily remedied.
\end{abstract}

\section{Introduction}

Within the aerodynamics community, the Spalart-Allmaras (SA) ${ }^{1}$ and the Menter shear stress transport (SST) $k-\omega$ turbulence models have been widely-used and trusted for Reynolds-averaged Navier-Stokes (RANS) computations for over a decade. The SA model is a one-equation model based primarily on empiricism and on dimensional analysis arguments. It works well for both attached and for many separated flows. It was one of the first field-equation models to gain wide acceptance into modern aerodynamic application CFD codes. The SST model combined the robustness of the $k-\omega$ turbulence model $^{3}$ near walls with the capabilities of the $k-\varepsilon$ model $^{4}$ away from walls (also removing the sensitivity to variations in farfield $\omega$ boundary conditions ${ }^{5}$ ). It also included the effects of turbulent shear stress transport through Bradshaw's assumption ${ }^{6}$ that the shear stress in a boundary layer is proportional to the turbulent kinetic energy. This "SST" modification was the key that gave the model its ability to predict separated flows much better than the standard $k-\omega$ form. Both the SA and SST models have been remarkably successful and stable (very few changes proposed or required) over the years.

Recently, Rumsey et al. ${ }^{7}$ showed that certain forms of the low Reynolds number two-equation $k-\varepsilon$ model could exhibit apparent transition behavior that is dependent on initial conditions and method of solution (such as different procedures of mesh sequencing). They determined the numerical reasons behind the long-recognized inconsistent transition prediction behavior of the $k-\varepsilon$ model (see, e.g., $\mathrm{Abid}^{8}$ ). The apparent laminar flow region predicted by the model upstream of transition was termed "pseudo-laminar" because, although the eddy viscosity was predicted to be near-zero (yielding laminar results for all intents and purposes), the behavior of the near-zero turbulent quantities were incorrect relative to each other in the laminar limit. The pseudo-laminar state could be reached in some regions of the flow solely because $k=\varepsilon=0$ was a stable solution to the equations under certain conditions, and not because of any physics built into the model.

CFD practitioners are generally aware that most transport turbulence models "transition" on their own."-11 The location of this apparent transition generally has no relationship with experimental results. In other words, in computed results there is usually a region of laminar flow followed by turbulence, even if a CFD code is run in fully-turbulent mode, with the turbulence equations active everywhere in the flow field. For the SA and SST models at reasonably high Reynolds numbers, this apparent transition location tends to occur very far upstream in most applications, and the models seem to avoid the aforementioned problems often seen with the $k-\varepsilon$ model.

* Senior Research Scientist, Computational Aerosciences Branch, Mail Stop 128, Associate Fellow AIAA, c.1.rumsey@ nasa.gov

This material is declared a work of the U.S. Government and is not subject to copyright protection in the United States.2006 
However, in light of the recent analysis of the $k-\varepsilon$ model, ${ }^{7}$ it is appropriate to apply a similar analysis to the SA and SST models in an effort to shed some light on their ability to produce undesirable apparent transition behavior. The main question to try to answer is whether there are circumstances for which the SA and SST models can yield inconsistent results in terms of the extent of laminar flow predicted. If present, such an inconsistency can have negative consequences when attempting to draw conclusions from computed results.

\section{Analysis of the Turbulence Models}

\section{A. Spalart-Allmaras}

The one-equation SA model is written in terms of the turbulence quantity $\tilde{\nu}$.

$$
\begin{aligned}
& \frac{\partial \tilde{\nu}}{\partial t}+u_{j} \frac{\partial \tilde{\nu}}{\partial x_{j}}=C_{b 1}\left(1-f_{t 2}\right) \tilde{S} \tilde{\nu}-\left[C_{w_{1}} f_{u^{\prime}}-\frac{C_{b 1}}{\kappa^{2}} f_{t 2}\right]\left(\frac{\tilde{\nu}}{d}\right)^{2} \\
& +\frac{1}{\sigma}\left[\frac{\partial}{\partial x_{j}}\left((\nu+\tilde{\nu}) \frac{\partial \tilde{\nu}}{\partial x_{j}}\right)+C_{b 2} \frac{\partial \tilde{\nu}}{\partial x_{i}} \frac{\partial \tilde{\nu}}{\partial x_{i}}\right]
\end{aligned}
$$

where

$$
\begin{gathered}
f_{t 2}=C_{t 3} \exp \left(-C_{t+1} \chi^{2}\right) \\
\tilde{S}=\Omega+\frac{\tilde{\nu} f_{v^{\prime}}}{\kappa^{2} d^{2}} \\
f_{v 1}=\frac{\chi^{3}}{\chi^{3}+C_{v 1}^{3}} \\
f_{v 2}=1-\frac{\chi}{1+\chi f_{v 1}} \\
f_{u^{\prime}}=g\left[\frac{1+C_{w 3}^{6}}{g^{6}+C_{w 3}^{6}}\right]^{1 / 6} \\
g=r+C_{w^{2}}\left(r^{6}-r\right) \\
r=\frac{\tilde{\nu}}{\tilde{S} \kappa^{2} d^{2}} \\
\chi=\frac{\tilde{\nu}}{\nu}
\end{gathered}
$$

The eddy viscosity is given by $\nu_{t}=\bar{\nu} f_{v^{\prime} 1}, \Omega$ is the magnitude of the vorticity, $d$ is the distance to the nearest wall, and the constants are: $C_{b 1}=0.1355, C_{b 2}=0.622,{ }_{i}=0.41, \sigma=2 / 3, C_{t 3}=1.2, C_{t 4}=0.5, C_{w 2}=0.3, C_{w 3}=2$, $C_{v 1}=7.1$, and $C_{w 1}=C_{b 1} / \kappa^{2}+\left(1+C_{b 2}\right) / \sigma$.

As noted in Spalart and Allmaras, ${ }^{1}$ the $f_{t: 2}$ term was an ad hoc numerical fix that makes $\tilde{\nu}=0$ a stable solution to the equations with a small basin of attraction (also note that its constants $C_{t, 3}$ and $C_{t 4}$ changed values between the AIAA paper reference and the subsequent journal article reference). The analysis below confirms this characteristic. The reason the model developers originally wanted this behavior was due to their use of a "trip function," $f_{t 1} \Delta U$, not described above but given in the original reference. Because the SA model without $f_{t 2}$ would often trip to turbulence upstream of where the numerical trip was set for transitional flows, Spalart and Allmaras invented the $f_{t 2}$ term to delay it. However, arguably most present-day users of SA do not employ the trip function, but rather run the model in fully-turbulent mode (or else force desired laminar regions by zeroing out the production term). In the Results section, potential undesirable consequences of the presence of the $f_{t 2}$ term for fully-turbulent computations will be shown.

For the first part of the analysis here, the homogeneous form of the one-equation model is used. As discussed in Rumsey et al., ${ }^{7}$ this is a useful approximation because the log-layer or equilibrium layer of a turbulent boundary layer has the same dynamical characteristics as a homogeneous flow. The dynamic similarity is exploited by treating the 
flow as "locally homogeneous." In other words, although the mean shear varies throughout a boundary layer, in the analysis the assumption is made of a locally fixed mean shear at a given location.

The homogeneous form of the SA equation is

$$
\frac{\partial \tilde{\nu}}{\partial t}=R
$$

where

$$
R=C_{b 1}\left(1-f_{t 2}\right) \tilde{S} \tilde{\nu}-\left[C_{w^{1}} f_{w}-\frac{C_{b 1}}{\kappa^{2}} f_{t 2}\right]\left(\frac{\tilde{\nu}}{d}\right)^{2}
$$

By inspection, it is clearly seen that $\tilde{\nu}=0$ is a critical point that satisfies the equation in the steady-state. The stability of this solution is investigated by solving for $\partial R / \partial \tilde{\nu}$ in the vicinity of $\tilde{\nu}=0$ :

$$
\begin{aligned}
& \frac{\partial R}{\partial \tilde{\nu}}=C_{b 1} \tilde{S}+C_{b 1} \tilde{\nu} \frac{\partial \tilde{S}}{\partial \tilde{\nu}}-C_{b 1} f_{t 2} \tilde{S}-C_{b 1} f_{t 2} \tilde{\nu} \frac{\partial \tilde{S}}{\partial \tilde{\nu}}-C_{b 1} \tilde{S} \tilde{\nu} \frac{\partial f_{t 2}}{\partial \tilde{\nu}} \\
& -2 \frac{C_{w^{1}}}{d^{2}} f_{w} \tilde{\nu}-\frac{C_{w 1}}{d^{2}} \tilde{\nu}^{2} \frac{\partial f_{w}}{\partial \tilde{\nu}}+2 \frac{C_{b 1}}{\kappa^{2} d^{2}} f_{t 2} \tilde{\nu}+\frac{C_{b 1}}{\kappa^{2} d^{2}} \tilde{\nu}^{2} \frac{\partial f_{t 2}}{\partial \tilde{\nu}}
\end{aligned}
$$

Using the chain rule and the fact that near $\tilde{\nu}=0: \tilde{S} \rightarrow \Omega, f_{v 1} \rightarrow 0, f_{v 2} \rightarrow 1, f_{t 2} \rightarrow C_{t 3}, r \rightarrow 0, g \rightarrow 0, f_{w} \rightarrow 0$, it can be shown that:

$$
\left.\frac{\partial R}{\partial \tilde{\nu}}\right|_{\tilde{\nu}=0} \rightarrow \Omega C_{b 1}\left(1-C_{t 3}\right)
$$

Because $C_{t 3}=1.2$, Eq. (13) is always negative, which means that the null critical point is always stable. Therefore, whenever $\tilde{\nu}$ gets in the vicinity of $\tilde{\nu}=0$, the solution to the partial differential equation may be attracted toward $\tilde{\nu}=0$.

This stability can be demonstrated numerically by solving for the instantaneous time rate-of-change of the turbulence quantity for a wide array of possible current state values. This is done by first writing Eqs. (10) - (11) in nondimensional form:

$$
\frac{\partial \tilde{\nu}^{\prime}}{\partial t^{\prime}}=C_{b 1}\left(1-f_{t 2}\right) \tilde{S}^{\prime} \tilde{\nu}^{\prime}-\left(\frac{M}{R e}\right)\left[C_{w 1} f_{w}-\frac{C_{b 1}}{\kappa^{2}} f_{t 2}\right]\left(\frac{\tilde{\nu}^{\prime}}{d^{\prime}}\right)^{2}
$$

where

$$
\tilde{S}^{\prime}=\Omega^{\prime}+\left(\frac{M}{R e}\right) \frac{\tilde{\nu}^{\prime} f_{v 2}}{\kappa^{2} d^{\prime 2}}
$$

(The quantity $r$ also requires a $(M / R e)$ factor: $r=(M / R e) \tilde{\nu}^{\prime} /\left(\tilde{S}^{\prime} \kappa^{2} d^{\prime 2}\right)$.) In these expressions, $\tilde{\nu}^{\prime}=\tilde{\nu} / \nu_{\text {ref }}$, $t^{\prime}=t a_{\text {ref }} / L_{\text {ref }}, d^{\prime}=d / L_{\text {ref }}$, and $\Omega^{\prime}=\Omega L_{\text {ref }} / a_{\text {ref }}$ (with $a_{\text {ref }}$ representing the reference speed of sound).

Next, a variety of values are input for the independent variables $\tilde{\nu}^{\prime}, d^{\prime} \sqrt{\operatorname{Re} / M}$, and $\Omega^{\prime}$. An example solution map is shown in Fig. 1. Here, setting $\Omega^{\prime}=1000$, a contour plot of $\partial \tilde{\nu}^{\prime} / \partial t^{\prime}$ is made for an array of $\tilde{\nu}^{\prime}$ and $d^{\prime} \sqrt{\operatorname{Re} / M}$ values. The solution map shows that $\partial \tilde{\nu}^{\prime} / \partial t^{\prime}<0$ for the region below and to the left of the neutral line (the solid line in the figure). This means that for an initial condition in this region the solution variable $\tilde{\nu}^{\prime}$ is driven lower as the numerical iteration procedure progresses. Above and to the right of the neutral line, $\partial \tilde{\nu}^{\prime} / \partial t^{\prime}>0$, which means that $\tilde{\nu}^{\prime}$ is driven higher. Thus, for $d^{\prime} \sqrt{R e / M}$ to the right of the dot in the figure, there are two possible stable solutions. The portion of the neutral line above the dot represents the turbulent solution to the homogeneous form of the SA equation. For a given $d^{\prime} \sqrt{\operatorname{Re} / M} \geq 0.09$ or so, the solution to the equation will converge to the corresponding $\tilde{\nu}^{\prime}$ on the stable neutral line, provided that the initial condition is somewhere above the unstable neutral line. Otherwise, the solution will converge to the degenerate laminar $\tilde{\nu}^{\prime}=0$ value.

Although not shown, for larger $d^{\prime} \sqrt{R e / M}$, the lower part of the neutral line approaches a constant value near $\tilde{\nu}^{\prime} \approx 0.6$, and the upper part of the neutral line continues upward and to the right. Also, for different values of $\Omega^{\prime}$, the general character of the solution map remains the same as that shown in the figure, but the specific contour shapes and levels shift.

This exercise using the homogeneous form of the SA equation is instructive, but not realistic. In the full form of the equation, advection and diffusion are locally active and play a significant role. These terms can have basically three effects on the solution map, when added to the right-hand-side of Eq. (14). First, if the sum of the terms is negative, 
then the general character of the solution map remains unchanged from the homogeneous map: only its levels and location of the neutral lines are different. Second, if the sum of the terms is positive and relatively large compared to the other terms in the equation, then the possible problem of laminar solution behavior goes away. An example solution map is shown in Fig. 2. For this map, $\Omega^{\prime}$ is again taken as 1000, but this time an assumed nondimensional advection plus diffusion contribution is taken to be a constant value of 10 , for demonstration purposes. The map shows that in this case there is only one neutral line; it is stable and corresponds to the turbulent solution. Any initial condition will converge to this turbulent solution. For fully turbulent flow, this situation is the desired one: the numerics are such that the solution always converges to the intended, unique value.

Third, very interesting non-unique behavior occurs if the sum of the right-hand-side advection plus diffusion is positive but not too large compared to the other terms. An example solution map is shown in Fig. 3. For demonstration purposes, $\Omega^{\prime}$ is again taken as 1000 , and nondimensional advection plus diffusion is taken to be a constant value of 3 . In this case, there are three distinct neutral lines. The lower line oriented horizontally is stable, and will be reached for any initial condition below the unstable neutral line. This lower stable neutral line has very low levels of $\tilde{\nu}^{\prime}$, yielding even smaller levels of eddy viscosity. Thus, convergence to this line represents a solution that behaves like laminar flow. It will be shown in the Results section that this solution behavior can cause the apparent transition location for a fully turbulent computation to vary noticeably with simple grid refinement.

\section{B. Menter SST $k-u^{\prime}$}

The two-equation SST model is written as follows:

$$
\begin{aligned}
\frac{D \rho k}{D t} & =P_{k}-\beta^{*} \rho \omega k+\frac{\partial}{\partial x_{j}}\left[\left(\mu+\frac{\mu_{t}}{\sigma_{k}}\right) \frac{\partial k}{\partial x_{j}}\right] \\
\frac{D \rho \omega}{D t} & =\frac{\gamma \rho}{\mu_{t}} P_{k}-\beta \rho \omega^{2}+\frac{\partial}{\partial x_{j}}\left[\left(\mu+\frac{\mu_{t}}{\sigma_{\omega}}\right) \frac{\partial \omega^{\prime}}{\partial x_{j}}\right]+2\left(1-F_{1}\right) \frac{\rho}{\sigma_{\omega 2} \omega} \frac{\partial k}{\partial x_{j}} \frac{\partial \omega}{\partial x_{j}}
\end{aligned}
$$

where

$$
\begin{gathered}
\mu_{t}=\frac{\rho a_{1} k}{\max \left(a_{1 \omega} \cdot \Omega F_{2}\right)} \\
P_{k}=\tau_{i j} \frac{\partial u_{i}}{\partial x_{j}} \quad\left(\approx \mu_{t} \Omega^{2} \text { in boundary layer flows }\right) \\
F_{1}=\tanh \left(\Gamma^{4}\right) \\
\Gamma=\min \left[\max \left(\frac{\sqrt{k}}{\beta^{*} \omega d}, \frac{500 \mu}{\rho \omega d^{2}}\right) \cdot \frac{4 \rho k}{\sigma_{\omega 2} \mathrm{CD}_{k \omega} d^{2}}\right] \\
\mathrm{CD}_{k \omega}=\max \left(\frac{2 \rho}{\sigma_{\omega 2} \omega} \frac{\partial k}{\partial x_{j}} \frac{\partial \omega}{\partial x_{j}} \cdot 10^{-20}\right) \\
F_{2}=\tanh \left(\Pi^{2}\right) \\
\Pi=\max \left(\frac{2 \sqrt{k}}{\beta^{*} \omega d}, \frac{500 \mu}{\rho \omega d^{2}}\right)
\end{gathered}
$$

and the parameters $\gamma, \sigma_{k}, \sigma_{\omega}$, and $\beta$ are calculated via:

$$
\begin{aligned}
\gamma & =F_{1} \gamma_{1}+\left(1-F_{1}\right) \gamma_{2} \\
\sigma_{k} & =F_{1} \sigma_{k 1}+\left(1-F_{1}\right) \sigma_{k 2} \\
\sigma_{\omega} & =F_{1} \sigma_{\omega 1}+\left(1-F_{1}\right) \sigma_{\omega 2} \\
\beta & =F_{1} \beta_{1}+\left(1-F_{1}\right) \beta_{2}
\end{aligned}
$$


and $\gamma_{1}=0.55317, \gamma_{2}=0.44035, \sigma_{k 1}=1.17647, \sigma_{k 2}=1, \sigma_{\omega 1}=2, \sigma_{\omega 2}=1.16822, \beta_{1}=0.075, \beta_{2}=0.0828$, $\kappa=0.41, a_{1}=0.31, \beta^{*}=0.09, \Omega$ is the magnitude of the vorticity, and $d$ is the distance to the nearest wall. Because many CFD codes use the approximation that $P_{k}=\mu_{t} \Omega^{2}$ (as recommended by Menter ${ }^{12}$ ), it is employed here as well.

As an initial step, we look briefly at the homogeneous form of the SST model. Because we are concerned primarily with transition and the inner part of the boundary layer, we perform this initial analysis with $F_{1}=F_{2}=1$. Therefore, the cross-diffusion term in Eq. (17) is zero. Nondimensionalizing the turbulence quantities via:

$$
\begin{aligned}
k^{\prime} & =k / a_{\text {ref }}^{2} \\
\omega^{\prime} & =\omega \mu_{\text {ref }} /\left(\rho_{\text {ref }} a_{\text {ref }}^{2}\right)
\end{aligned}
$$

and then, for convenience, further normalizing these variables, along with time, via:

$$
\begin{aligned}
k^{*} & =\Omega^{\prime} k^{\prime} \\
\omega^{*} & =\frac{\omega^{\prime}}{\Omega^{\prime}}\left(\frac{R e}{M}\right) \\
t^{*} & =\Omega^{\prime} t^{\prime}
\end{aligned}
$$

the homogeneous form of SST inside the boundary layer can be written:

$$
\begin{aligned}
\frac{\partial k^{*}}{\partial t^{*}} & =\min \left(\frac{k^{*}}{\omega^{*}}, a_{1} k^{*}\right)-\beta^{*} k^{*} \omega^{*} \\
\frac{\partial \omega^{*}}{\partial t^{*}} & =\gamma_{1}-\beta_{1} \omega^{* 2}
\end{aligned}
$$

Similar to the SA model, a degenerate solution satisfies these equations in the steady state: $k^{*}=0, \omega^{*}=\sqrt{\gamma_{1} / \beta_{1}}$. This value of $\omega^{*}$ is less than $1 / a_{1}$ for the values of the given constants, so $a_{1} k^{*}<k^{*} / \omega^{*}$, and the stability of the degenerate solution can be found from the eigenvalues of the coefficient matrix of the linear system (Eqs. (34) and (35)):

$$
J=\left[\begin{array}{cc}
a_{1}-\beta^{*} \omega^{*} & -\beta^{*} k^{*} \\
0 & -2 \beta_{1} \omega^{*}
\end{array}\right]
$$

Plugging in the degenerate solution, the eigenvalues are found to be real with one positive and one negative: this indicates that the solution is a saddle point. ${ }^{7}$ Generally, a saddle point is not stable in practice because orbits approach the critical point along one eigenvector, but then recede along the eigenvector associated with the unstable solution. Thus, this analysis suggests that the SST model should not be attracted to the degenerate solution.

However, as with the SA model, it is important to also consider the effects of the advective and diffusive terms in the SST model in order to truly begin to understand its dynamical behavior. The easiest way to do this is to perform an actual computation, and monitor the values of all the terms as the solution progresses. This has been done, and detailed results will be shown in the Results section. However, for the purposes of analysis and attempting to get a picture of the overall $k^{*}-\omega^{*}$ solution space, we make the assumption that the dominant turbulent transport effects act over a distance that represents the length scale characterizing the large turbulent eddies, ${ }^{13}$ given by $\ell=k^{3 / 2} / \varepsilon=k^{1 / 2} / \omega$. Then, the nondimensional advection and diffusion terms in the turbulent region can be approximated by:

$$
\begin{array}{rlc}
k \text {-eqn advection } & : & U_{j}^{\prime} \frac{\partial k^{\prime}}{\partial x_{j}^{\prime}} \sim \mathcal{O}\left(\frac{k^{\prime}}{\ell^{\prime}}\right) \sim \mathcal{O}\left(k^{\prime 1 / 2} \omega^{\prime}\right)=\left(C_{k, A}\right) k^{\prime 1 / 2} \omega^{\prime} \\
k-\text { eqn diffusion } & : & \frac{\partial}{\partial x_{j}^{\prime}}\left[\frac{\mu_{t}^{\prime}}{\sigma_{k}} \frac{\partial k^{\prime}}{\partial x_{j}^{\prime}}\right] \sim \mathcal{O}\left(\frac{k^{\prime 2}}{\omega^{\prime} \ell^{\prime 2}}\right) \sim \mathcal{O}\left(k^{\prime} \omega^{\prime}\right)=\left(C_{k, D}\right) k^{\prime} \omega^{\prime} \\
\omega-\text { eqn advection } & : & U_{j}^{\prime} \frac{\partial \omega^{\prime}}{\partial x_{j}^{\prime}} \sim \mathcal{O}\left(\frac{\omega^{\prime}}{\ell^{\prime}}\right) \sim \mathcal{O}\left(k^{\prime-1 / 2} \omega^{\prime 2}\right)=\left(C_{\omega, A}\right) k^{\prime-1 / 2} \omega^{\prime 2} \\
\omega-\text { eqn diffusion } & : & \frac{\partial}{\partial x_{j}^{\prime}}\left[\frac{\mu_{t}^{\prime}}{\sigma_{\omega}} \frac{\partial \omega^{\prime}}{\partial x_{j}^{\prime}}\right] \sim \mathcal{O}\left(\frac{k^{\prime}}{\ell^{\prime 2}}\right) \sim \mathcal{O}\left(\omega^{\prime 2}\right)=\left(C_{\omega, D}\right) \omega^{\prime 2}
\end{array}
$$




$$
\omega \text {-eqn cross diffusion }: \quad 2\left(1-F_{1}\right) \frac{\rho^{\prime}}{\sigma_{\omega 2} \omega^{\prime}} \frac{\partial k^{\prime}}{\partial x_{j}^{\prime}} \frac{\partial \omega^{\prime}}{\partial x_{j}^{\prime}} \sim \mathcal{O}\left(\frac{k^{\prime}}{\ell^{\prime 2}}\right) \sim \mathcal{O}\left(\omega^{\prime 2}\right)=\left(C_{\omega, C D}\right) \omega^{\prime 2}
$$

where $\mu_{t}^{\prime}=\mu_{t} / \mu_{\text {ref }}$, and $C_{k . A}, C_{k . D}, C_{\omega, 4}, C_{\lrcorner, D}$, and $C_{\omega . C D}$ are (unknown) coefficients that can be determined at instantaneous locations and times in a particular flow field via numerical computations. Also, $U_{j}^{\prime}$ represents the (nondimensional) mean flow velocity components, which are taken as locally constant and are absorbed into the coefficients $C_{k, A}$ and $C_{\omega, A}$. Employing the normalizations from Eqs. (31) - (33), the inhomogeneous equations can be approximated as:

$$
\begin{aligned}
& \frac{\partial k^{*}}{\partial t^{*}}=\min \left(\frac{k^{*}}{\omega^{*}}, \frac{a_{1} k^{*}}{F_{2}}\right)+\left(C_{k, D}-\beta^{*}\right) k^{*} \omega^{*}+\left(C_{k, A}\right) k^{* 1 / 2} \omega^{*} \\
& \frac{\partial \omega^{*}}{\partial t^{*}}=\gamma+\left(C_{\omega, D}+C_{\omega \cdot C D}-\beta\right) \omega^{* 2}+\left(C_{\omega, A}\right) k^{*-1 / 2} \omega^{* 2}
\end{aligned}
$$

(with $F_{1}$ and $F_{2}$ no longer assumed to be 1 ). These equations are admittedly very crude, because of the approximations made in Eqs. (37) - (41). In fact, analysis of the actual advection and diffusion terms from CFD results at several locations in the bounday layer confirmed that the coefficients $C_{k, A}$, etc., do not come out to be constant. However, the length scale $\ell=k^{3 / 2} / £$ yielded a fairly reasonable approximation that was much better than other choices. The solution to Eqs. (42) and (43) can be found. It is the intersection of the $k^{*}$-nullcline, given by:

$$
\begin{array}{rlrl}
\omega^{*} & =\left(\frac{1}{3^{*}-\left(C_{k, A}\right) k^{*-1 / 2}-C_{k, D}}\right)^{1 / 2} & \omega^{*}>\frac{F_{2}}{a_{1}} \\
\omega^{* *}=\frac{a_{1}}{F_{2}\left(\beta^{*}-\left(C_{k, A}\right) k^{*-1 / 2}-C_{k, D}\right)} & \omega^{*}<\frac{F_{2}}{a_{1}}
\end{array}
$$

and the $\omega^{*}$-nullcline, given by:

$$
\omega^{*}=\left(\frac{\gamma}{\beta-\left(C_{\omega, A}\right) k^{*-1 / 2}-C_{\omega, D}-C_{\omega, C D}}\right)^{1 / 2}
$$

The analytic form of the eigenvalues associated with this solution is very complicated, and does not yield a clear conclusion regarding the stability of the solution. However, it is easy to numerically find the eigenvalues for a point in the turbulent boundary layer of a typical computation. In all cases investigated to date, the solution has turned out to be a saddle point. An example phase-plane portrait is shown in Fig. 4. This figure shows the $k^{*}$ - and $\omega^{*}$ nullclines using coefficients obtained at a point in a converged turbulent boundary layer where nondimensional eddy viscosity $\mu_{t}^{\prime}=26.47524$. In this flow field, $R e=1 \times 10^{7}$ and $M=0.3$. The converged solution at this point (circled in the figure) has a value of $k^{\prime}=0.7312424 \times 10^{-3}, \omega^{\prime}=0.2386361 \times 10^{-4}$, and nondimensional vorticity magnitude $\Omega^{\prime}=265.3249$. Thus $k^{*}=0.1940168$ and $\omega^{*}=2.998036$. Other coefficients obtained from the numerical solution were: $C_{k, D}=-0.3368563 \times 10^{-2}, C_{k, A}=-0.4419278 \times 10^{-2}, C_{\omega, D}+C_{\omega, C D}=0.9705072 \times 10^{-2}$, $C_{\omega, A}=0.1760321 \times 10^{-2}, F_{1}=0.9879624$, and $F_{2}=0.9999944$. The figure also shows the local trajectories (defined by $\partial k^{*} / \partial t^{*}$ and $\partial \omega^{*} / \partial t^{*}$ ) of the turbulence variables in phase-space.

It is clear from the figure that the solution is a saddle point: solution trajectories approach from above and below, but recede in both directions along the $\omega^{*}$-nullcline. This is somewhat surprising, because at a saddle point an orbit is usually attracted at first but repelled later on. This would seem to imply that the SST model is not strictly stable. However, nonlinear and nonlocal effects were not accounted for in the analysis, and it turns out that these act to maintain stability at the solution point. For example, using a numerical experiment from the example in Fig. 4, when $k^{*}$ at the solution point was perturbed slightly to the left, then the local spatial $k^{*}$ derivatives also changed, yielding different local advective and diffusive terms which temporarily shifted the intersection point of the nullclines even further to the left; as a result the solution was driven back to the right, toward its original position. In any case, SST certainly is numerically stable in practice.

Although not shown here, the standard $k \cdot-w$ model $^{3}$ yields a phase-plane portrait very similar to that of SST, with its solution also appearing as a saddle point. Thus, its behavior is expected to be similar.

Now the following question arises: what are the effects of this model's seemingly tenuous linearized-solution stability on its apparent transition behavior? The answer is not entirely clear from this analysis, but in the Results section some details will be shown that will hopefully shed some light on this question. 


\section{Numerical Method}

The computer code CFL3D ${ }^{14}$ solves the three-dimensional, time-dependent compressible RANS equations with an upwind finite-volume formulation (it can also be exercised in two-dimensional mode of operation for 2-D cases). Upwind-biased third-order spatial differencing is used for the inviscid terms, and viscous terms are centrally differenced. The code originally solved the thin-layer form of the equations (in each coordinate direction), but the full Navier-Stokes terms (i.e., cross-derivative terms) have recently been added. All solutions shown below use the full Navier-Stokes terms, although thin-layer has also been employed and makes almost no difference for the particular cases run.

The CFL3D code is advanced in time with an implicit approximate factorization method. The implicit derivatives are written as spatially first-order accurate, which results in block tridiagonal inversions for each sweep. However, for solutions that utilize Roe flux-difference splitting, ${ }^{15}$ the block tridiagonal inversions are further simplified using a diagonal algorithm with a spectral radius scaling of the viscous terms.

The turbulence models, including SA and SST, are solved uncoupled from the mean flow equations using implicit approximate factorization. Their advective terms can be solved using either first-order or second-order upwind differencing, with first-order the default for the code.

\section{Results}

In order to demonstrate inconsistent apparent transition behavior of the SA and SST models, it was necessary to perform computations on very fine grids. Easily noticeable inconsistencies did not show up using coarser grids. The model problem chosen was the computation over the front upper $45 \%$ of a NACA 0012 airfoil, at $M=0.3$, $R e=10^{7}$ based on total chord length, and $\alpha=0^{\circ}$. Fully-turbulent computations were performed, which means that the turbulence model equations were active everywhere in the flow field. The freestream eddy viscosity was set using a typical value ${ }^{16}$ of turbulence Reynolds number $R_{T, \infty}=k_{\infty}^{2} /\left(\nu_{\infty} \varepsilon_{\infty}\right)=0.1$, yielding $\mu_{t, \infty}=0.009$. For the SA model, this was achieved using $\tilde{\nu}_{\infty}^{\prime}=1.341946$. It will be shown below that for the SA model, the freestream turbulence level chosen can be an important consideration. For the SST model, the freestream turbulent quantities used were: $k_{\infty}^{\prime}=9 \times 10^{-9}$ and $\omega_{\infty}^{\prime}=1 \times 10^{-6}$.

The finest grid was $769 \times 1025$, with coarser grids created by successively removing every other grid point from the next finest level. Thus the coarser grid sizes were: $385 \times 513,193 \times 257,97 \times 129$, and $49 \times 65$. The grids had a farfield extent of 50 chords, and the minimum normal spacing at the wall yielded an average $y+$ level of 0.05 for the $769 \times 1025$ grid, 0.1 for the $385 \times 513$ grid, 0.2 for the $193 \times 257$ grid, 0.4 for the $97 \times 129$ grid, and 0.8 for the $49 \times 65$ grid.

A picture of the coarsest grid is shown in Fig. 5. Note that this coarsest level is on a par with typical grid resolution used for many turbulent CFD computations over airfoils and wings. For boundary conditions, the part of the grid coming into the nose of the airfoil was set as a symmetry plane, the airfoil body used no-slip adiabatic wall conditions, the downstream exit plane used extrapolation, and the farfield (upstream and above the airfoil) used a farfield Riemann invariant condition. For most of the computations, each grid level was run by restarting from the previous coarse grid solution, as is typically done for full-approximation scheme (FAS) multigrid simulations. ${ }^{17}$ All results were converged 5 - 6 orders of magnitude on each grid (the final density residual dropped to $10^{-10}-10^{-11}$ ).

\section{A. Spalart-Allmaras}

Fig. 6 shows computed surface skin friction coefficients on the airfoil using SA for each of the grids. As the grid was refined, each successive solution produced a larger "laminar region" prior to going turbulent. Only on the finest $769 \times$ 1025 grid did the transition prediction appear to stop changing significantly. For the coarsest grid, the nondimensional eddy viscosity first exceeded 1.0 in the boundary layer near $x / c=0.0044$. For successively finer grids, the location was $0.0056,0.0099,0.0111$, and 0.0112 , respectively. As will be shown next, this apparent later transition on the finer grids is a numerical artifact related to the existence of the laminar-behavior stable neutral line for this turbulence model.

Solution maps can be created for this realistic computation by monitoring the various independent quantities during the course of the solution's progress. In this case, the quantities were recorded during the solution on the $193 \times 257$ grid, at a point in the boundary layer near $x / c=0.00694$, where $d^{\prime} \sqrt{R e / M}=0.1157$. At this position, the converged solution on the $97 \times 129$ grid was turbulent (i.e., the peak nondimensional eddy viscosity level - located somewhat further from the wall at this location - exceeded 1), but during iterations on the $193 \times 257$ grid this location became 
laminar.

Fig. 7 shows the descent of the $\tilde{\nu}^{\prime}$ quantity at this location from near 3.9 initial value to a converged value of 0.205 . (These correspond to nondimensional eddy viscosity levels of about 0.48 and $4 \times 10^{-6}$, respectively). This solution on the $193 \times 257$ was restarted from the converged solution on the $97 \times 129$ grid at multigrid cycle number 8001 . Next, by using the actual instantaneous values for $\Omega^{\prime}$ and nondimensional advection plus diffusion at this location, the solution maps were plotted at multigrid cycle numbers 8940,9120 , and 11000 (at cycle 8940: $\tilde{\nu}^{\prime}=3.1064, \Omega^{\prime}=5365.3$, and advection plus diffusion $=-748.8$; at cycle $9120: \bar{\nu}^{\prime}=2.313, \Omega^{\prime}=5399.4$, and advection plus diffusion $=-1137.4$; at cycle 11000: $\tilde{\nu}^{\prime}=0.205, \Omega^{\prime}=5500.3$, and advection plus diffusion $=24.5$ ). These maps are shown in Figs. 8,9, and 10. At cycle 8940, the solution map shows that the location of the current value of $\tilde{\nu}^{\prime}$ (indicated by the open circle) lies slightly above and to the left of the stable neutral line in the map space. Thus, its value is being driven lower with successive iterations. As its value decreases, the local advection and diffusion also change significantly such that the stable neutral line is driven further to the right (Fig. 9). Finally, at convergence (Fig. 10), the final value of $\tilde{\nu}^{\prime}$ lies on the stable neutral line representative of a laminar-behavior solution.

Because two stable attractors exist, it is possible to obtain different solutions with SA depending on numerical factors such as initial condition (I.C.) and method of solution. An example is shown for the $97 \times 129$ grid in Fig. 11 . The result labeled "I.C. \#1" is the same result on this grid level shown earlier in Fig. 6. The result labeled "I.C. \#2" was obtained from an initial condition using a converged result on the $193 \times 257$ grid interpolated onto the coarser level, then re-iterated to convergence. Two different apparent transition locations result: the first near $x / c=0.0056$ and the second near $x / c=0.0097$.

The numerically-induced laminar behavior of the SA model is easily avoided in two different ways. The first method, as suggested by Spalart, ${ }^{10}$ is to employ higher levels of freestream turbulence. For example, using $\bar{\nu}_{\propto}^{\prime}=3$ $\left(\mu_{t, \infty}^{\prime}=0.2104\right)$, the problem of inconsistent transition was eliminated. as shown in Fig. 12. The apparent transition location remained near the airfoil leading edge (near $x / c=0.004$ at this Reynolds number) for all grid levels.

The earlier SA analysis also suggests a second method for avoiding the problem. By setting the constant $C_{t: 3}=0$, the right-hand side of Eq. (13) remains always positive, and the null critical point is unstable ( $\tilde{\nu}=0$ is not an attractor). The same NACA 0012 airfoil case was run with $\mu_{t, \infty}^{\prime}=0.009$ and $C_{t 3}=0$. Although not shown, results again show consistent behavior, and skin friction results appear identical to those shown in Fig. 12.

\section{B. Menter SST $k-\omega$}

The same NACA 0012 case was run using the SST model in fully turbulent mode. Results are shown in Fig. 13, and are similar to those using SA: as the grid was refined, the apparent transition location moved further aft. Even on the finest $769 \times 1025$ grid, the location still appeared to be influenced by the grid density. For the coarsest grid, the nondimensional eddy viscosity first exceeded 1.0 in the boundary layer near $x / c=0.0054$. For successively finer grids, the location was $0.0062,0.0076,0.0086$, and 0.0089 , respectively. It should be noted that, unlike the SA model above and the $k-\varepsilon$ model reported in Rumsey et al., ${ }^{7}$ SST did not appear to exhibit sensitivity to initial conditions or method of solution. In other words, a given grid always gave the same result.

The dynamics of the SST solution behavior near the leading edge region was investigated by monitoring various quantities during the solution progress on the $193 \times 257$ grid, at a point in the boundary layer near $x / c=0.00694$ (distance from the wall was $d^{\prime} \sqrt{R e / M}=0.2285$ ). At this streamwise location, the solution on the $97 \times 129$ grid was turbulent (peak nondimensional eddy viscosity exceeded 1 in the boundary layer), but during iterations on the $193 \times 257$ grid the peak eddy viscosity dropped below 1 .

Fig. 14 shows the changes in $\mu_{t}^{\prime}, k^{*}$, and $\omega^{*}$ through iteration to convergence. The value of $\omega^{*}$ changed relatively little, but $k^{*}$ decreased by nearly $75 \%$, causing a similar dramatic decrease in eddy viscosity. At multigrid cycle number 8500 , the phase-plane portrait in Fig. 15 shows that the unconverged values of $k^{*}$ and $\omega^{*}$ (indicated by the open circle) lie to the left of the intersection point of the nullclines. Thus, the solution in phase-space continues to be driven to the left. As it moves, the nullclines also change, but their intersection remains to the right of the solution until convergence is reached. Finally, at convergence (Fig. 16), the solution and the intersection of the nullclines coincide, as they must.

Unlike SA, the SST model continued to exhibit inconsistent transition results even when the freestream turbulence level was raised to levels above 1 . For example, Fig. 17 shows surface skin friction coefficients with $\mu_{t, x}^{\prime}=1.294$ $\left(k_{\infty}^{\prime}=1.294 \times 10^{-f^{-6}}\right)$. Compared with results in Fig. 13, these results using higher freestream turbulence show less grid influence, but the influence is still visible nonetheless.

From these examples, it seems evident that the SST model does indeed exhibit a sensitivity of its predicted transition location to grid density, but this sensitivity is not due to a null critical point acting as an additional attractor. Rather, there is only one possible solution, and the model dynamical behavior itself (near the area where turbulence 
initiates) appears to be particularly sensitive to small changes induced by different numerical resolution. One of the reasons for this sensitivity may be the fact that, in a linear sense, the solution is a saddle point in $k^{*}-\omega^{*}$ phase-space. No simple cure was found to remedy this problem. It seems that it would be more desirable to have the system of turbulence equations exhibit strong stability, such as that shown in Fig. 18. Here, in this hypothetical plot (not based on any real model), the solution trajectories are all strongly attracted in toward the fixed point.

As mentioned earlier, although the analysis and examples were done here specifically for SST, similar results are exhibited by the general form of the $k-\omega$ model as well.

\section{Conclusions}

In conclusion, both the Spalart-Allmaras and the Menter SST $k$ - $\omega$ turbulence models were shown to have the undesirable characteristic that, for fully turbulent computations, their apparent transition location can vary with grid density. As the grid was refined for an example airfoil computation, an apparent laminar region near the nose of the airfoil grew larger. In the SA model this behavior was due to a feature purposefully added to the original model in conjunction with a special trip function, which many people employing the model do not even use. Solution maps were used to show that this feature can cause convergence to a laminar-behaving fixed point. Because two stable fixed points exist, even a given grid can produce non-unique results regarding where transition initiates. It was also shown that consistent fully turbulent $S A$ results can be achieved in practice by either using higher freestream turbulence levels $\left(\tilde{\nu}_{\infty}^{\prime}=3\right.$ or greater), or by setting the constant $C_{t 3}$ in the model to 0 .

The SST model's dynamical behavior (near the area where turbulence initiates) appears to be particularly sensitive to small changes induced by changes in numerical resolution. Thus, its apparent transition location change with grid refinement seems to be a fundamental property of the $k-\omega$ model itself, and is not easily remedied. Raising freestream turbulence levels did not fix the problem. Phase-plane portraits, which included the nullclines of the $k-\omega$ equations, were used to demonstrate the SST solution behavior. This model does not appear to suffer from the problem of possible non-uniqueness on a given grid.

It is important to note that the problems illustrated in this paper do not appear to be very significant in practice. For example, the transition location movement appears to be fairly small for airfoils relative to the airfoil chord. In other words, generally these two turbulence models tend to transition near enough to the leading edge of airfoils and wings so that the overall solution remains globally consistent. However, this may be problem and/or Reynolds number dependent. In any case, running the SA model with $\tilde{\nu}_{\infty}^{\prime} \geq 3$ for computations that do not use the trip function appears to be a good idea. Setting $C_{t 3}=0$ is also a viable choice, but may be less desirable from the point of view of model backward compatibility, because it is possible that the model behavior in and near separation could also be affected by this change. For $k-\omega$ models, including SST, users should be aware of the apparent transition-location dependence on grid density. In particular, as computers become more powerful and finer grids are more routinely employed, the problem behavior may manifest itself to a greater degree.

\section{Acknowledgments}

The author gratefully acknowledges T. B. Gatski of the Laboratoire d'Etudes Aerodynamiques, Universite de Poitiers, B. A. Pettersson Reif of the Norwegian Defence Research Establishment, and P. R. Spalart of the Boeing Company for their comments and advice.

\section{References}

\footnotetext{
${ }^{1}$ Spalart, P. R., and Allmaras, S. R., "A One-Equation Turbulence Model for Aerodynamic Flows," La Recherche Aerospatiale, No. 1, 1994, pp. 5-21; also AIAA Paper 92-0439, Jan. 1992.

${ }^{2}$ Menter, F. R., "Two-Equation Eddy-Viscosity Turbulence Models for Engineering Applications," AIAA Journal, Vol. 32, No. 8, 1994, pp. $1598-1605$.

${ }^{3}$ Wilcox, D. C., Turbulence Modeling for CFD, Ist ed., DCW Industries, La Canada, 1994.

${ }^{4}$ Jones, W. P. and Launder, B. E., "The Prediction of Laminarization with a Two-Equation Model of Turbulence," Int. J. Heat Mass Transfer. Vol. 15, 1972, pp. 301-314.

${ }^{5}$ Menter, F. R., "Influence of Freestream Values on $k$ - $\omega$ Turbulence Model Predictions," AlAA Journal, Vol. 30, No. 6, 1992, pp. $1657-1659$.

${ }^{6}$ Bradshaw, P., Ferriss, D. H., and Atwell, N. P., "Calculation of Boundary-Layer Development Using the Turbulent Energy Equation," J. Fluid Mech., Vol. 28, Part 3, 1967,pp. 593-616.

${ }^{7}$ Rumsey, C. L., Pettersson Reif, B. A., and Gatski, T. B., "Arbitrary Steady-State Solutions with the K-Epsilon Model," AIAA J., accepted for publication.
} 
${ }^{8}$ Abid, R., "Evaluation of Two-Equation Turbulence Models for Predicting Transitional Flows," Int. J. Engng Sci., Vol. 31, No. 6, 1993, pp. $831-840$

${ }^{9}$ Carlson, J. R., “Applications of Algebraic Reynolds Stress Turbulence Models, Part 1: Incompressible Flat Plate," J. Propulsion and Power, Vol. 13, No. 5, 1997, pp. 610-619.

${ }^{10}$ Spalart, P. R., "Trends in Turbulence Treatments," AIAA Paper 2000-2306, June 2000

${ }^{11}$ Rumsey, C. L. and Biedron, R. T., "Computation of Flow over a Drag Prediction Workshop Wing/Body Transport Configuration using CFL3D," NASA/TM-2001-211262, December 2001.

${ }^{12}$ Menter, F. R., “Improved Two-Equation $k$ - $\omega$ Turbulence Models for Aerodynamic Flows,” NASA TM-103975, October 1992.

${ }^{13}$ Pope, S. B., Turbulent Flows, Cambridge University Press, Cambridge, U.K., 2000, p. 200

${ }^{14}$ Krist, S. L., Biedron, R. T., and Rumsey, C. L., "CFL3D User’s Manual (Version 5.0)," NASA TM-1998-208444, June 1998.

${ }^{15}$ Roe, P. L., "Approximate Riemann Solvers, Parameter Vectors, and Difference Schemes," J. Comp. Phys., Vol. 43, 1981, pp. 357-372.

${ }^{16}$ Baldwin, B. S. and Barth, T. J., "A One-Equation Turbulence Transport Model for High Reynolds Number Wall-Bounded Flows," NASA TM-102847, August 1990.

${ }^{17}$ Brandt, A., "Multi-Level Adaptive Computations in Fluid Dynamics," AIAA Paper 79-1455, July 1979

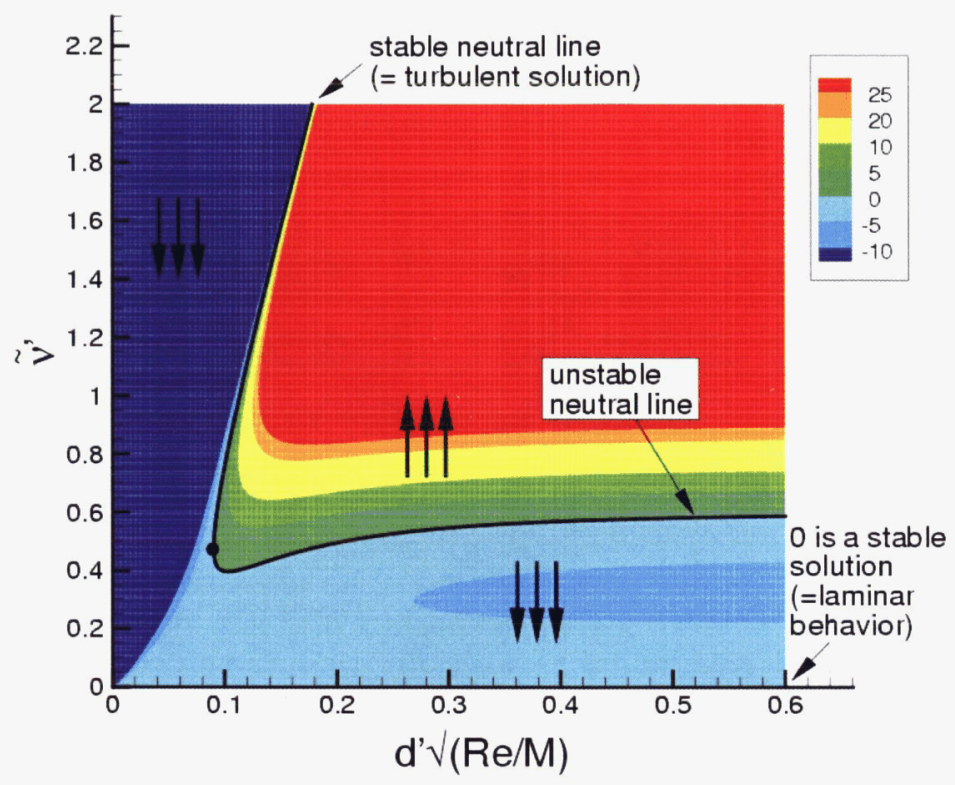

Figure 1. Homogeneous SA solution map of $\partial \tilde{\nu}^{\prime} / \partial t^{\prime}$ contours for a range of initial conditions for $\tilde{\nu}^{\prime}$ and $d^{\prime} \sqrt{R e / M}$; the solid line represents $\partial \tilde{\nu}^{\prime} / \partial t^{\prime}=0 ; \Omega^{\prime}=1000$. Initial values below and to the left of the neutral line are driven lower, and initial values above and to the right of the neutral line are driven higher. 


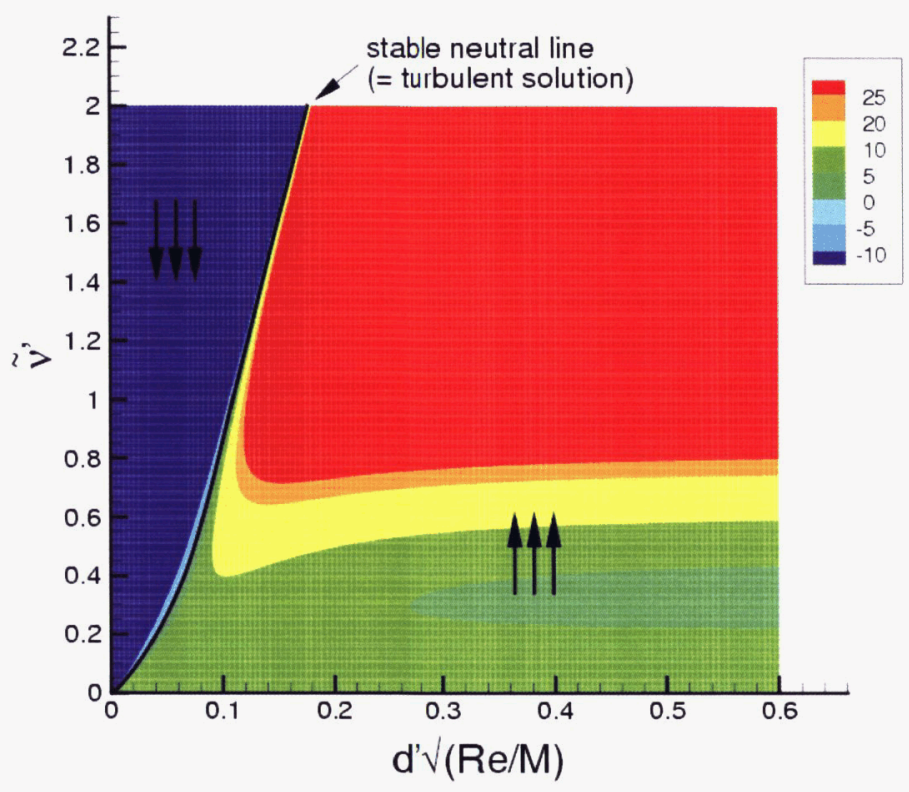

Figure 2. Inhomogeneous SA solution map of $\partial \tilde{\nu}^{\prime} / \partial t^{\prime}$ contours for a range of initial conditions for $\tilde{\nu}^{\prime}$ and $d^{\prime} \sqrt{R e / M}$; the solid line represents $\partial \tilde{\nu}^{\prime} / \partial t^{\prime}=0 ; \Omega^{\prime}=1000$; advection plus diffusion contribution taken to be 10 .

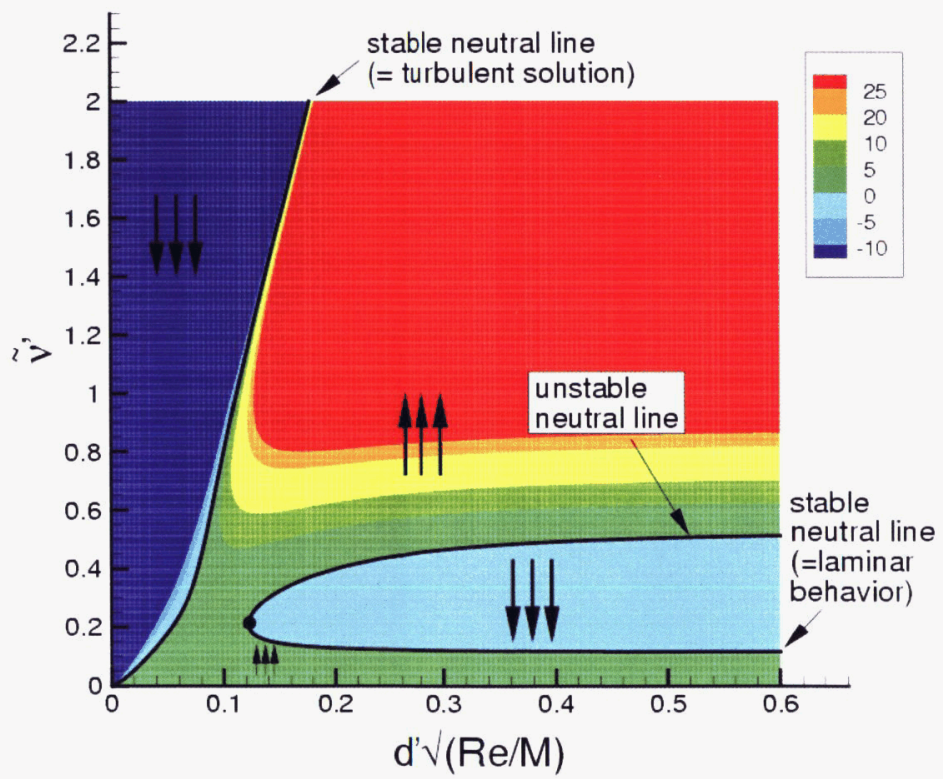

Figure 3. Inhomogeneous SA solution map of $\partial \tilde{\nu}^{\prime} / \partial t^{\prime}$ contours for a range of initial conditions for $\tilde{\nu}^{\prime}$ and $d^{\prime} \sqrt{R e / M}$; the solid line represents $\partial \tilde{\nu}^{\prime} / \partial t^{\prime}=0 ; \Omega^{\prime}=1000$; advection plus diffusion contribution taken to be 3 . 


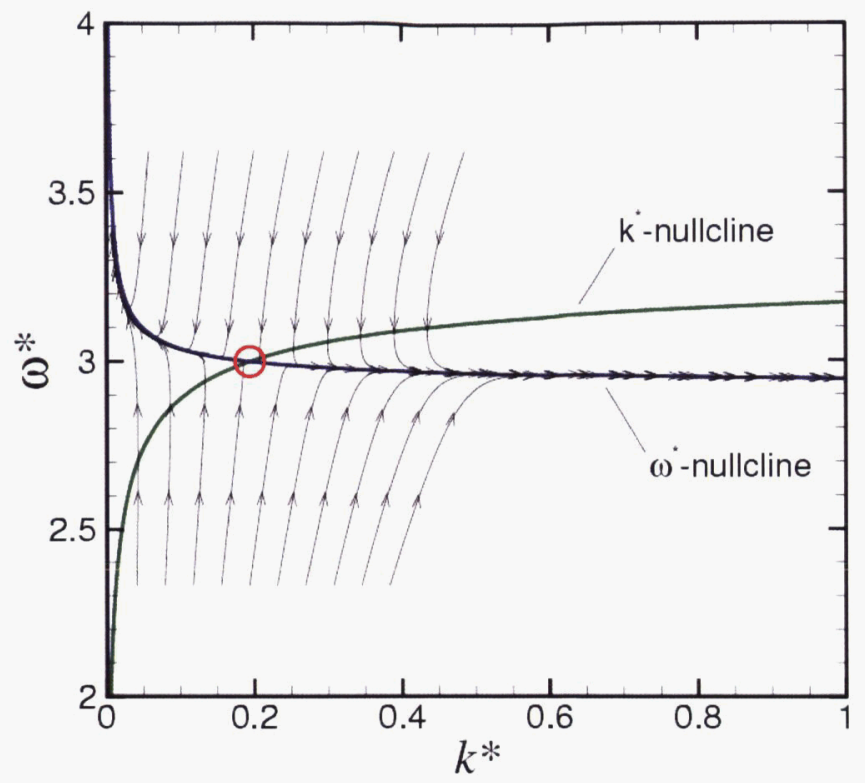

Figure 4. Inhomogeneous phase-plane portrait of the SST model in $k^{*}$ and $\omega^{*}$ space for a typical turbulent solution point (circled).

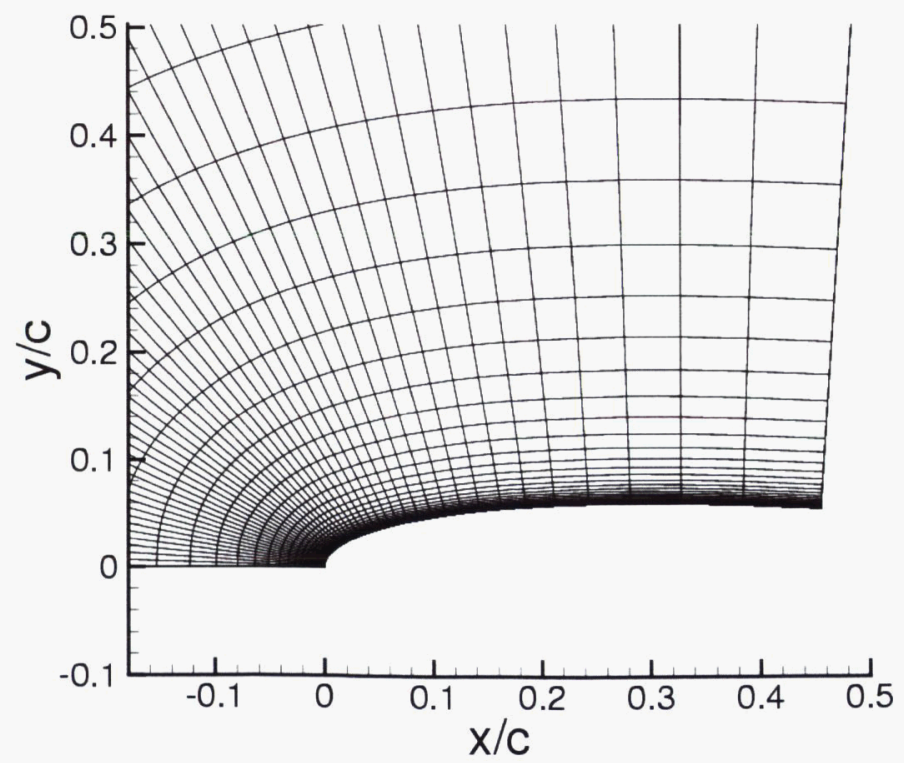

Figure 5. Portion of coarsest NACA 0012 grid, $49 \times 65$. 


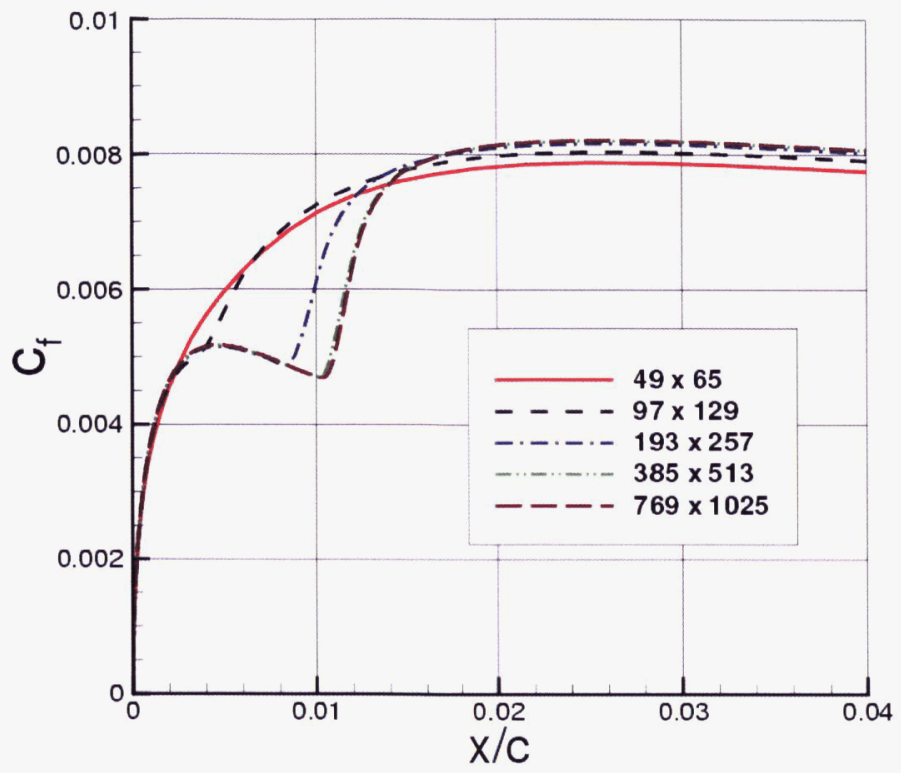

Figure 6. Computed surface skin friction coefficient for the NACA 0012 airfoil on various grids, SA model with $\mu_{t, \infty}^{\prime}=0.009$.

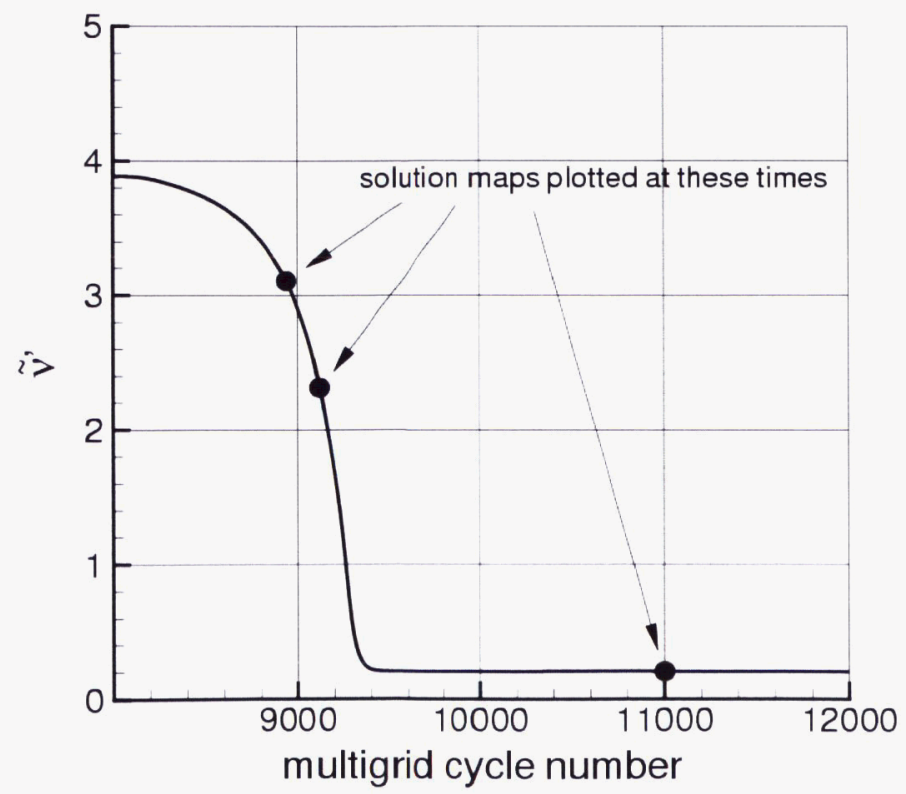

Figure 7. Convergence of $\tilde{\nu}^{\prime}$ at specific location in the boundary layer on $193 \times 257$ grid, SA model with $\mu_{t, \infty}^{\prime}=0.009$; solution maps will be plotted for the three times indicated in this figure. 


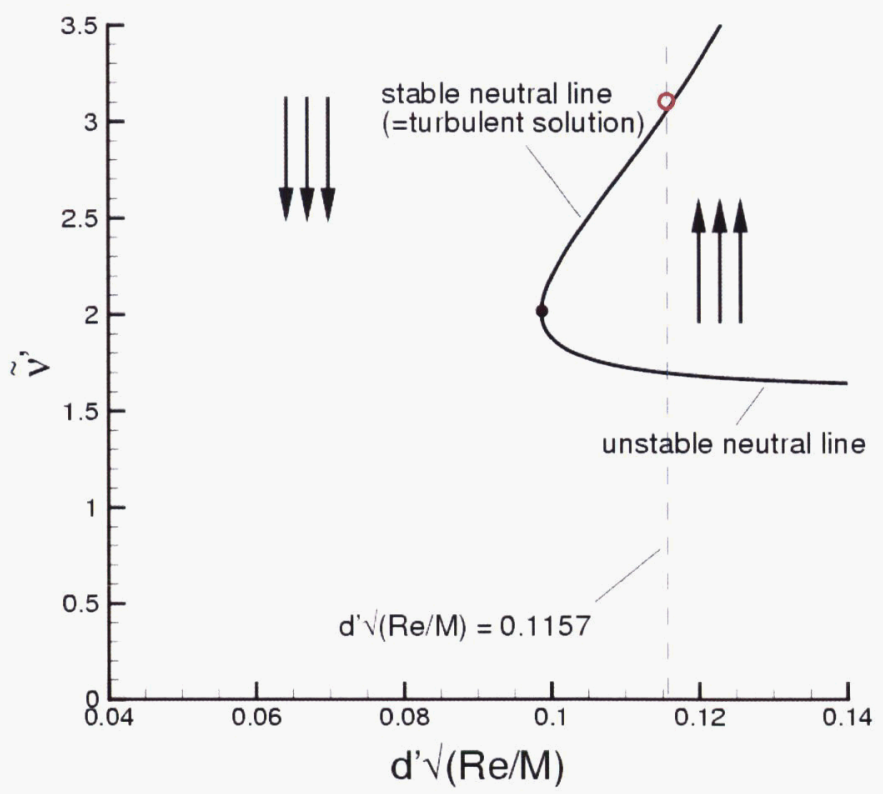

Figure 8. SA solution map for NACA 0012 case on $193 \times 257$ grid at a specific point in the boundary layer near $x / c=0.00694$, at multigrid cycle number 8940. Open circle indicates the particular (unconverged) solution at this time.

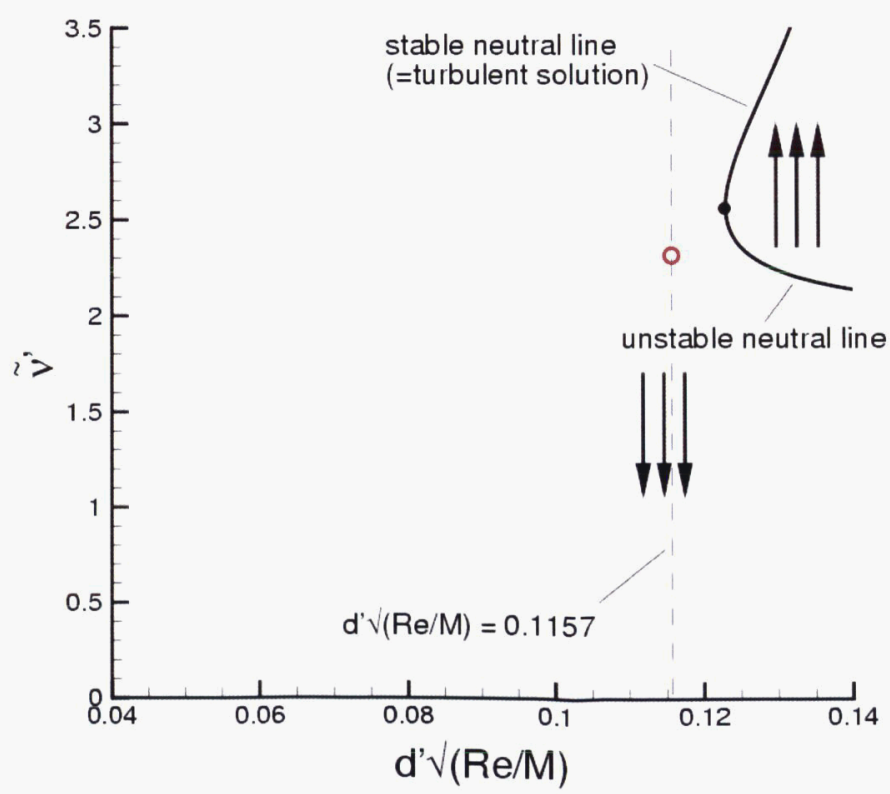

Figure 9. SA solution map for NACA 0012 case on $193 \times 257$ grid at a specific point in the boundary layer near $x / c=0.00694$, at multigrid cycle number 9120 . Open circle indicates the particular (unconverged) solution at this time. 


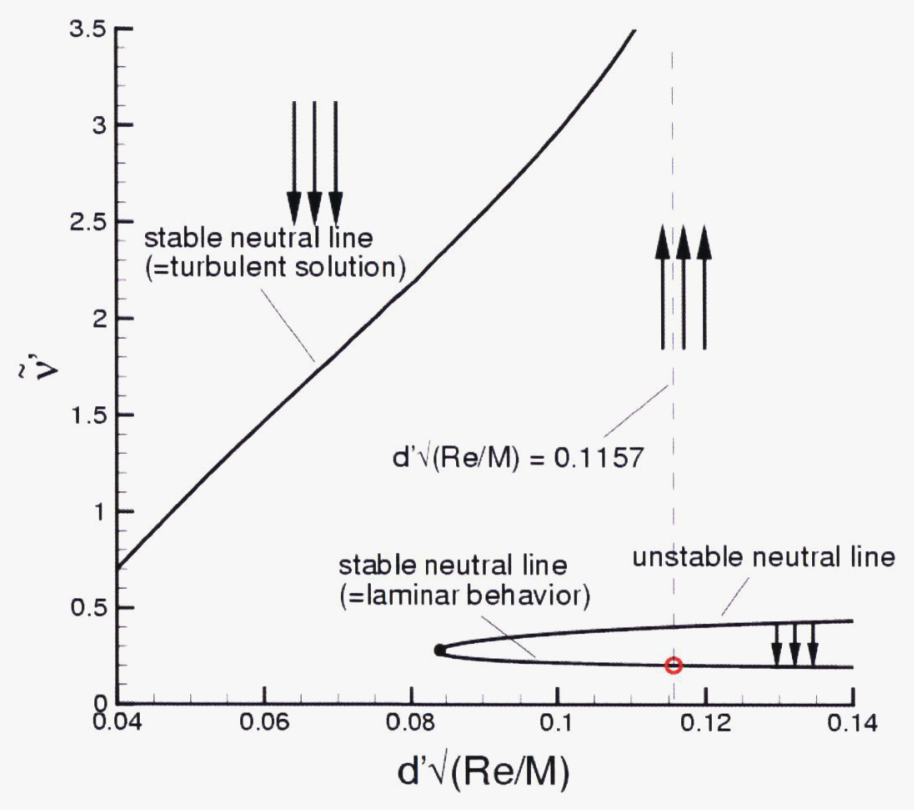

Figure 10. SA solution map for NACA 0012 case on $193 \times 257$ grid at a specific point in the boundary layer near $x / c=0.00694$, at multigrid cycle number 11000. Open circle indicates the particular (converged) final solution.

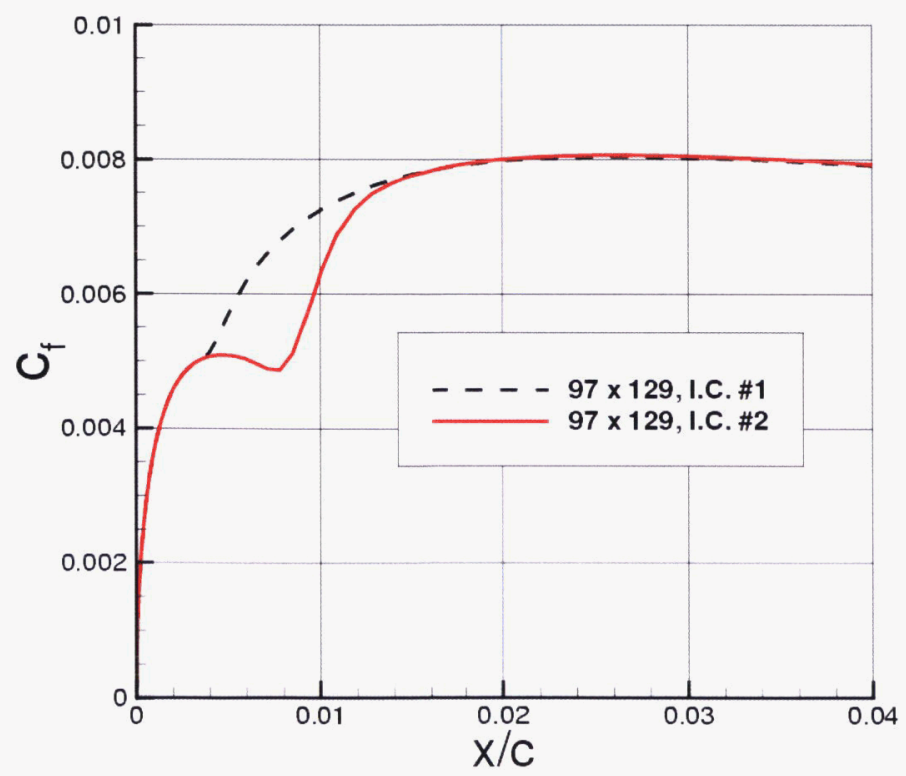

Figure 11. Computed surface skin friction coefficient for the NACA 0012 airfoil on the $97 \times 129$ grid, SA model with $\mu_{t, \infty}^{\prime}=0.009$, with two different initial conditions, demonstrating converged solution non-uniqueness. 


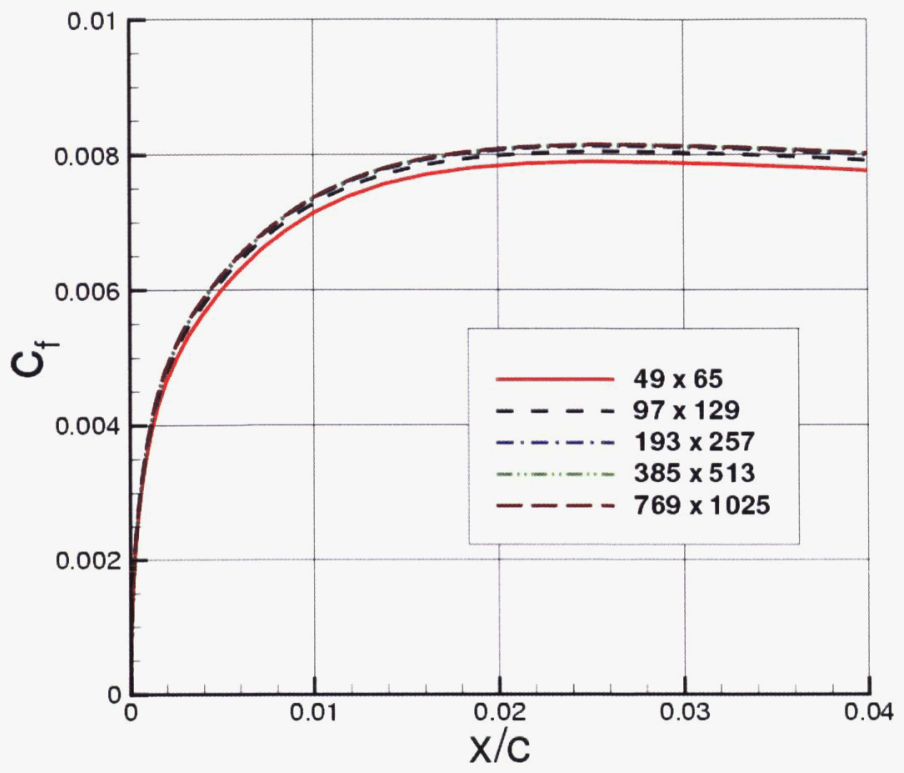

Figure 12. Computed surface skin friction coefficient for the NACA 0012 airfoil on various grids, SA model with $\mu_{t, \infty}^{\prime}=0.2104$.

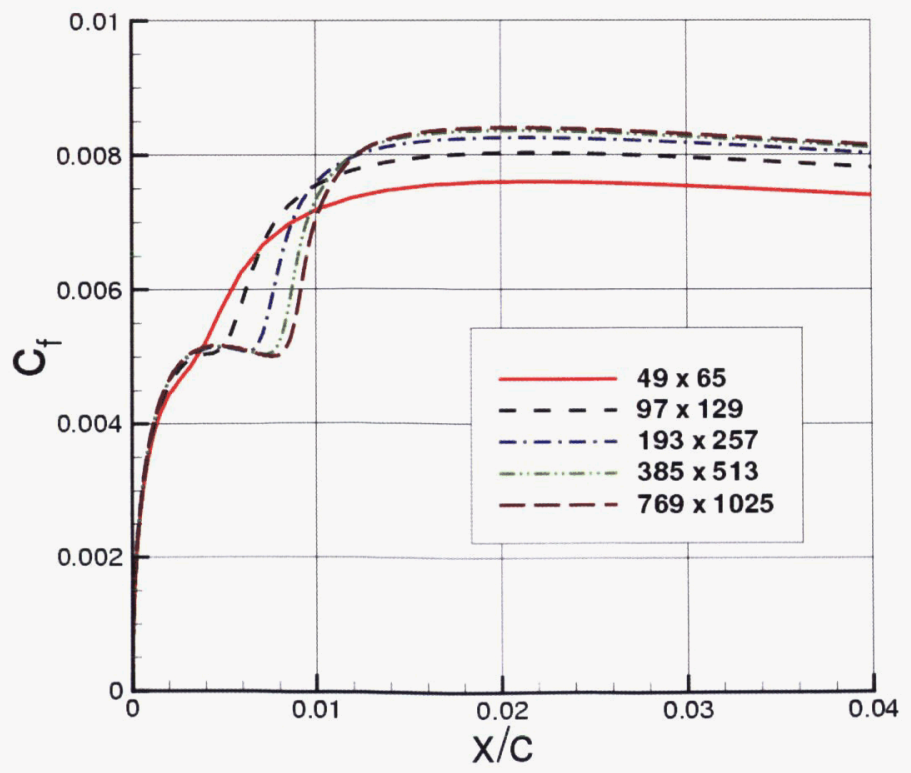

Figure 13. Computed surface skin friction coefficient for the NACA 0012 airfoil on various grids, SST model with $\mu_{t, \infty}^{\prime}=0.009$. 


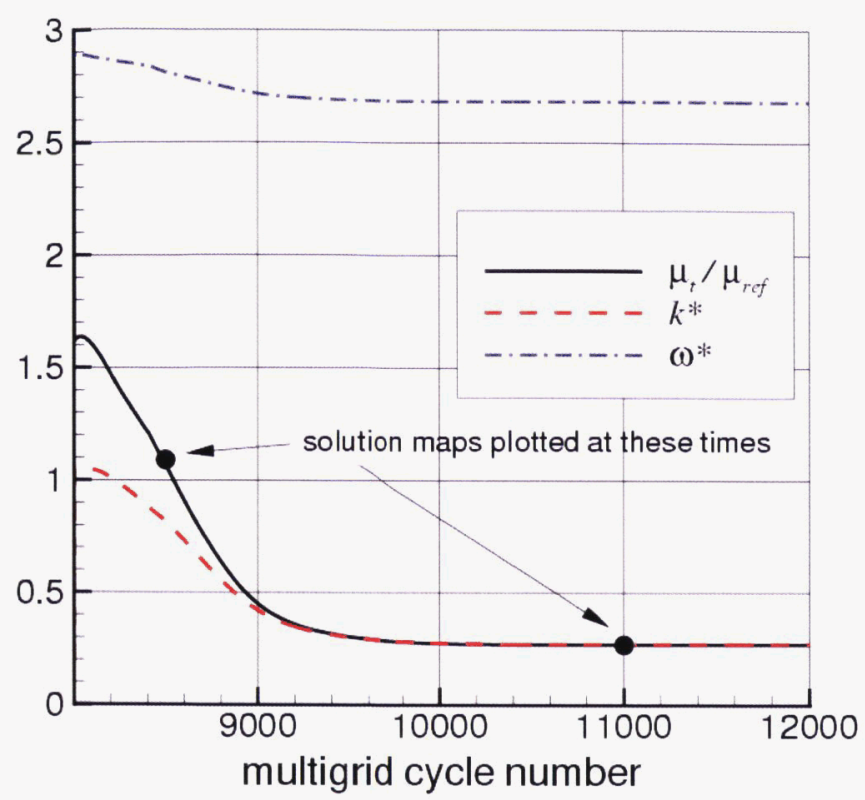

Figure 14. Convergence of $\mu_{t}^{\prime}, k^{*}$, and $\omega^{*}$ at specific location in the boundary layer on $193 \times 257$ grid, SST model with $\mu_{t, \infty}^{\prime}=0.009$; phase-plane portraits will be plotted for the two times indicated in this figure.

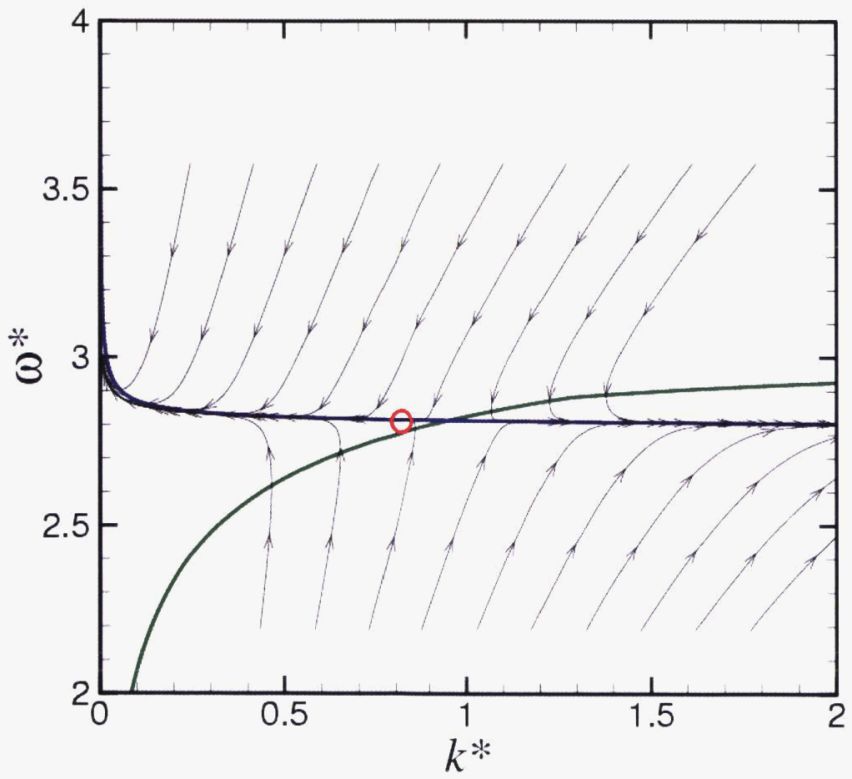

Figure 15. SST phase-plane portrait for NACA 0012 case on $193 \times 257$ grid at a specific point in the boundary layer near $x / c=0.00694$, at multigrid cycle number $\mathbf{8 5 0 0}$. Open circle indicates the particular (unconverged) solution at this time. 


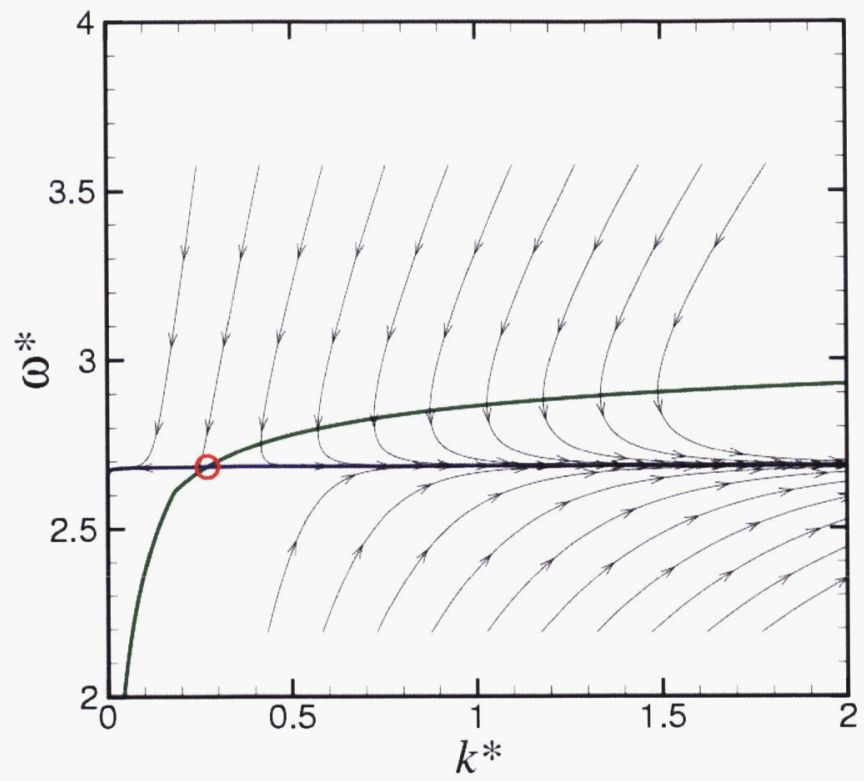

Figure 16. SST phase-plane portrait for NACA 0012 case on $193 \times 257$ grid at a specific point in the boundary layer near $x / c=0.00694$, at multigrid cycle number 11000 . Open circle indicates the particular (converged) final solution.

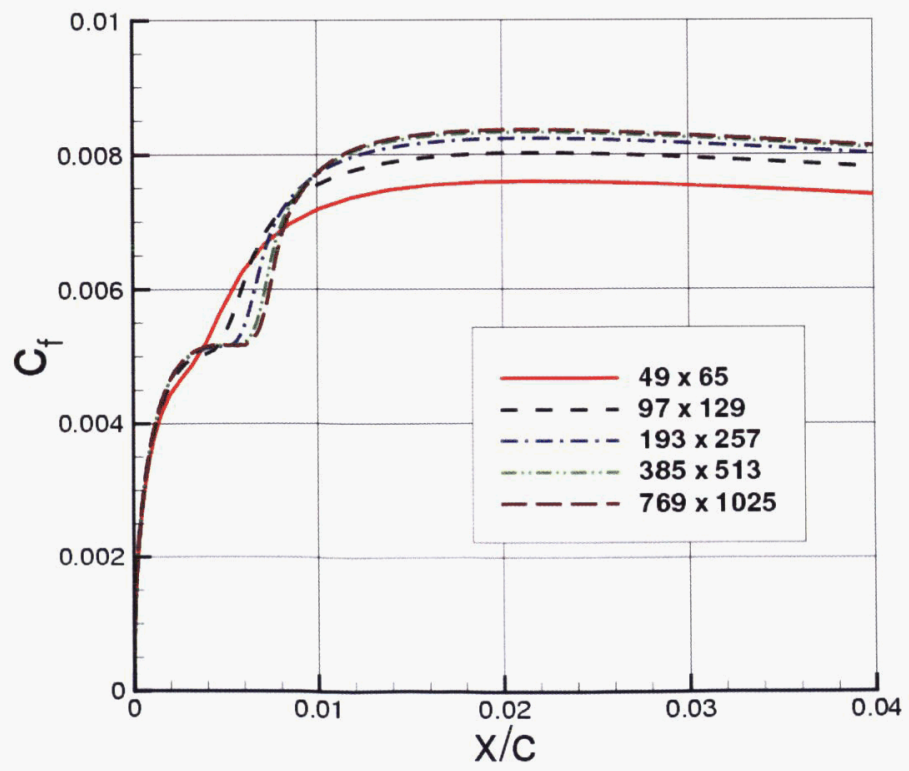

Figure 17. Computed surface skin friction coefficient for the NACA 0012 airfoil on various grids, SST model with $\mu_{t, \infty}^{\prime}=1.294$. 


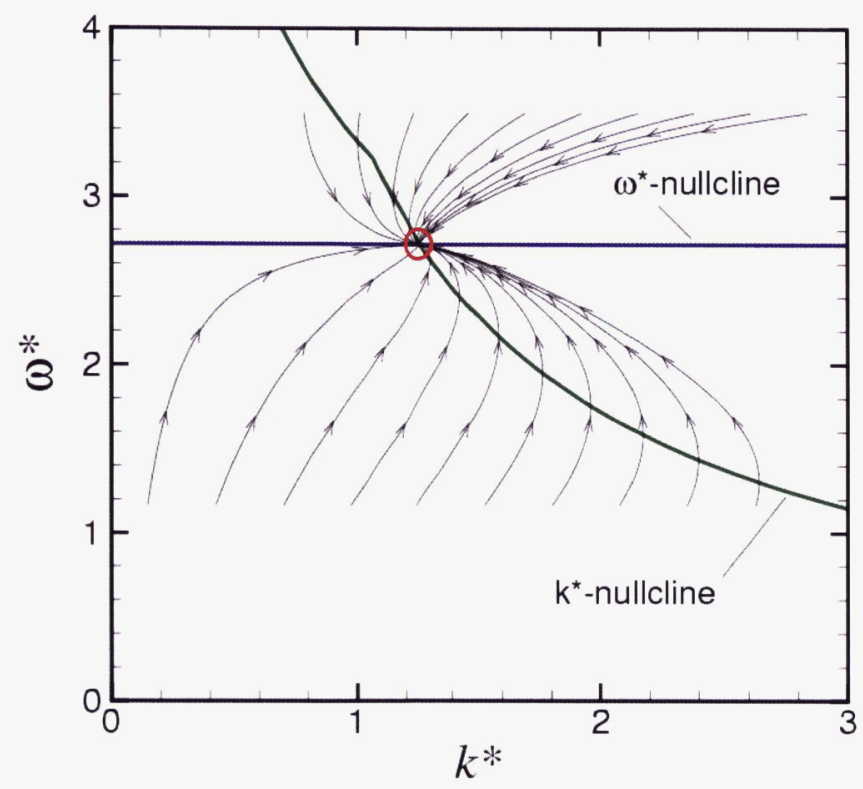

Figure 18. A hypothetical phase-plane portrait for which the $k^{*}$-nullcline crosses the $\omega^{*}$-nullcline in the opposite direction than in Fig. 4 , yielding a stable solution point rather than a saddle point. 